\title{
Acute Kidney Injury due to Russell's Viper Envenomation: First Documented Case in Nepal
}

\author{
B Bartaula, B Shah, N Bhurtel, SK Sharma
}

Department of Internal Medicine, BPKIHS, Dharan, Nepal

\begin{abstract}
Snake bite, is a significant public health problem and medical emergency in tropical countries including Nepal. Acute renal failure is one of the devastating complications of Russell's viper envenoming which may cause death of patient in the absence of prompt and adequate management. Treatment with anti-venom and hemodialysis can salvage the patient. Although Russell's viper is widely distributed in Nepal, AKI due to Russell's viper envenoming was not reported previously from Nepal.
\end{abstract}

DOI Name:

https://doi.org/10.3126/jaim.v7i2.23492

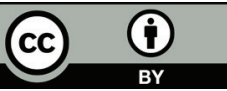

This work is licensed under a Creative Commons Attribution 3.0 Unported License.

\section{Introduction}

Snake bite, is a significant public health problem and medical emergency in tropical countries including Nepal and is frequently associated with significant morbidity and mortality. About 20,000 snake bite cases including 1000 deaths due to venomous snake bite occur in Nepal every year. ${ }^{1}$ The highest figures reported from Asia, so far, come from a community-based survey conducted in South-East Nepal in 2002. Among 22 species of highly venomous snakes found in Nepal, most of the deaths are inflicted by neurotoxic envenoming. ${ }^{2}$

Russell's viper (Doboia russelii) envenoming is known to cause hematotoxicity, acute kidney injury (AKI), neurotoxicity as well as rhabdomyolysis. AKI may also result from the direct action of some venoms and associated hypotension due to bleeding. ${ }^{3}$ Although Russell's viper is widely distributed in low land Nepal, the proven envenoming from Nepal is scarce. To the best of our knowledge no documented case of AKI has been reported till date from Nepal. We report a case of AKI due to Russell's viper bite envenomation.

\section{Case Report:}

A 28 year old female, farmer from eastern Terai with no comorbidities was bitten by a snake on her left hand cutting grass around 8:00 am. The bite was quite painful and she noticed the snake. Her hand was swollen and there was bleeding from the injured site. She was rushed to the nearby local health care center where they reached after six hours of bite. During the transport, she developed two episodes of hematemesis and one episode of malena.

In the local center she received 10 vials of polyvalent anti-snake venom. Subcutaneous adrenaline was given to prevent anaphylaxis to antivenom. Multiple blisters and the swelling of entire hand was noted.

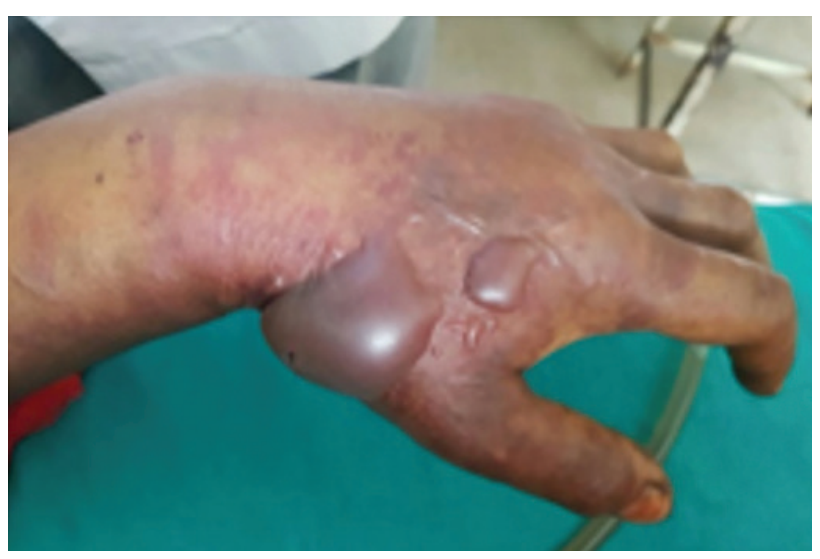

Patient was hemodynamically unstable with hypotension and tachycardia.

Further, she had two more episodes of hematemesis and one episode of melena in the health center. She was still hypotensive with evidence of shock. Her Blood Pressure was $60 / 40 \mathrm{mmHg}$ which was not corrected after infusion of 3 liters of crystalloid solution and had cold clammy peripheries and low volume pulse. Gradually her urine output decreased. Her bleeding time was noted to be more than 20 minutes. Due to persistence of shock and bleeding she was referred to our center for further evaluation and management.

\section{Correspondence Author}

B. Bartaula

Assistant Professor, Department Of Internal

Medicine, BPKIHS, Dharan, Nepal

Email: Bijaya83bartaula@gmail.com 
First seen in emergency of our hospital, she was found to be in shock, however she was conscious, oriented to time, place and person. During her stay in emergency she again developed two episodes of hematemesis. Her blood Pressure was 60/20 $\mathrm{mmHg}$, pulse $-110 / \mathrm{min}$ and of low volume and had cold clammy peripheries. Bite marks on finger was prominent with bleeding from bite site. Her hand was swollen, red and tender. There were multiple blisters in her hand. She had also developed conjunctival chemosis and sub-conjunctival hemorrhage.

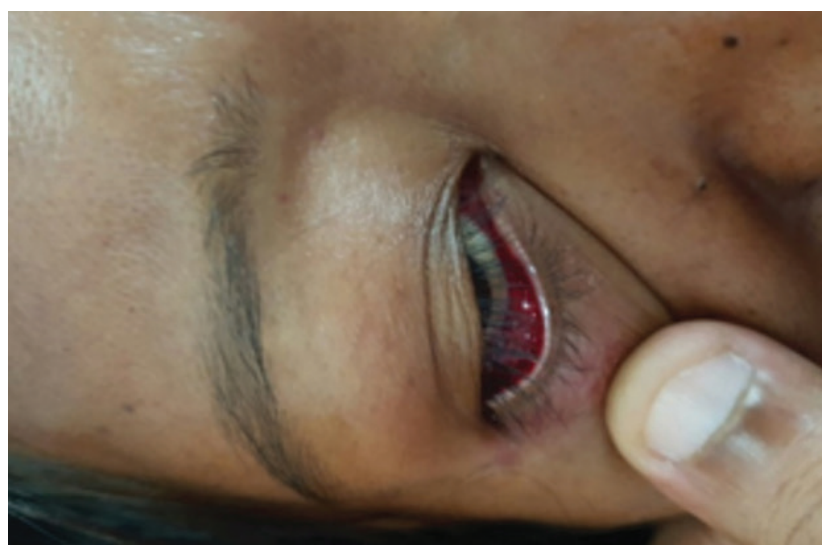

On urinary catheterization gross hematuria was noted. Clinically, cardiovascular, central nervous system and respiratory system examination had normal findings.

Her biochemical reports revealed hemoglobin-7.2gm/dl, total leucocyte count- 12300/cumm, Platelets- 35000/cumm, Urea$153 \mathrm{mg} / \mathrm{dl}$ Creatinine-3.1mg/dl, Na- $132 \mathrm{mmol} / \mathrm{L}, \mathrm{K}-3.4 /$ $\mathrm{mmol} / \mathrm{L}$, INR- 2.0 and creatinine kinase- 1957 units. ECG, chest $\mathrm{x}$-ray and echocardiography did not reveal any abnormality.

Patient initially received fluid challenge with isotonic saline in the view of hypovolemic shock and was advised to arrange blood and fresh frozen plasma as she had ongoing bleeding. She also received vasopressor noradrenaline and steroid. She further received 5 vials of antivenom bolus. Antivenom was continued till whole blood clotting time (WBCT) and Bleeding time (BT) returned to normal. After 24 hours of antivenom her WBCT became coagulable. Patient was deemed to be in state of acute DIC and was transfused 3 pints of PCV, 2 pints of FFP and 2 pints of PRP subsequently.

She continued to receive intravenous fluids (4 ltrs) daily for two days in view of rhabdomyolysis, a possibility for kidney injury. Her urine output did not improve and serum urea and creatinine kept rising. Intravenous fluid was restricted on $3 \mathrm{rd}$ day due to fluid overload and persistent oligoanuria. She was initiated on haemodialysis on third day of admission. On 9 th day of admission, after 4 sessions of dialysis, she started passing urine and went into diuretic phase of AKI. Patient's electrolytes and renal function was serially monitored and patient was discharged on 15 th day of admission. Renal biopsy was also planned on discharge. At the time of discharge her serum urea- $96 \mathrm{Cr}-3.6 / \mathrm{dl}$, bleeding time- 2 mins, clotting time -7 mins. INR 1.24 , serum Na- $134 / \mathrm{mmol} / \mathrm{L}$, K- $3.4 / \mathrm{mmol} / \mathrm{L}$, Hb- $9.3 \mathrm{gm} / \mathrm{dl}$ TLC 7400/cumm, Platelets494000/cumm, Creatinine kinase 222 units.

\section{Discussion :}

Russell's viper envenomation may result in AKI by multiple mechanisms. Some of the postulations are

1. Hypovolemic shock secondary to bleeding disorder,

2. Rhabdomyolysis leading to direct kidney injury or secondary to obstructive uropathy from myoglobin.
3. Similarly, acute tubular necrosis secondary to ischemia and disseminated intravascular coagulation due to snake venom is another mechanism of AKI.

As our patient had bleeding disorder, high creatinine kinase, and shock all the above mentioned mechanisms could have possibly played a role in our patient too.. Similar postulation was also put forward by Waikhom et al. ${ }^{3}$ Various authors had put forward the mechanisms of AKI in Russell's viper envenoming. Acute kidney injury due to direct nephrotoxic effect of snake venom was proposed by Singh et al, although there was no convincing evidence to implicate this. Hadler and Brazil documented renal tubular lesions to be due to the effect of lysolecithin.

Another study by Chugh, documented the the presence of fibrin thrombi in the renal microvasculature and in the glomerular capillaries. ${ }^{4}$ The findings of microangiopathic hemolytic anemia and thrombocytopenia in patients with cortical necrosis strongly suggest DIC as the culprit for the renal lesions of snake-bite induced cortical necrosis.

AKI due to snakebite is a common complication of hemotoxic snakebite and may need renal supportive therapy which may not be available in resource poor setting. Prompt recognition of envenoming and administration of antivenom is lifesaving. However, antivenom itself may not be sufficient to treat this devastating condition as seen in our patient. Therefore, timely referral of patients with anticipated AKI to center with renal support therapy is needed. To the best of our knowledge, this is the first case report of AKI related to Russell's viper envenoming in Nepal. As our patient was asymptomatic for glomerulopathy before the envenomation and kidney biopsy did not account for the other etiology for AKI, we attributed AKI in our patient to Russell's viper envenoming complications.

\section{Conclusion}

Acute renal failure is one of the devastating complications of Russell's viper envenoming which may cause death of patient in absence of prompt and adequate management. Treatment with anti-venom and hemodialysis salvaged our patient. Although Russell's viper is widely distributed in Nepal AKI due to Russell's viper envenoming was not reported previously from Nepal.

\section{References:}

1. World Health Organization, 1987. Zoonotic disease control: baseline epidemiological study on snake-bite treatment and management. Wkly Epidemiol Rec 42: 319-320. Sharma SK (2015). Snakebite and Acute Kidney Injury: We Must do Better! Indian Pediatrics 52:70-71.

2. Sharma SK, Chappuis F, Jha N, et al. Impact of snake bites and determinants of fatal outcomes in southeastern Nepal. Am J Trop Med Hyg. 2004 Aug; 71(2):234-8.

3. Waikhom R, Sapam R, Patil K, et al. Herpes labialis in patients with Russell's viper bite and acute kidney injury: a single center experience. Am J Trop Med Hyg. 2011; 84(6):1016-20.

4. Chugh KS. Snake-bite-induced acute renal failure in India. Kidney international 1989; 35(3), pp. 891-907. 